\title{
Fuzzy Assessment for Affective and Cognitive Computing in Intelligent E-learning Systems
}

\author{
Mohamed El Alami \\ Université Abdelmalek Essaâdi \\ Ecole Nationale de Sciences \\ Appliquées, Tanger (Maroc)
}

\author{
Fernando de Arriaga \\ Polytechnic University of Madrid, School of \\ Telecom. Eng., \\ Ciudad Univ., Madrid (Spain)
}

\begin{abstract}
A new generation of Intelligent E-Learning systems (ILS) has been designed and implemented by means of the construction of an agent factory, NEOCAMPUS2, able to produce "ad hoc" multi-agent structures for developing ILS. The paper describes some fuzzy assessment techniques suitable to be used with affective and cognitive computing and gives some results obtained with DYNAMICS2, a NEOCAMPUS2 spin-off ILS. The positive results, so far obtained, encourage the introduction of those fuzzy techniques in all NEOCAMPUS2 spin-off systems dealing with affective and cognitive computing, now under way.
\end{abstract}

\section{General Terms}

E-learning systems, fuzzy techniques, logic models, student's evaluation.

\section{Keywords}

Affective computing, intelligent e-learning, multiagent systems, fuzzy logic, cognitive modelling, student's assessment

\section{INTRODUCTION}

During the last years the analysis and design of Intelligent Tutoring Systems (ITS) have suffered important changes caused by new impacts of Artificial Intelligence and Information and Communication Technologies in general. The incorporation of Multi-Agent Architectures in the beginnings of the 90's improved a great deal the ITS capabilities allowing the cooperation of optics, models and ideas from Cognitive Psychology and other disciplines.

On the other hand, in the field of educational sciences, new approaches to learning, known as student centred education [1], [2], [3] have set up new methods and techniques for education based on active, collaborative and cooperative learning environments. The incorporation of those approaches to ITS has produced a new generation of intelligent systems, called ILS, in which the emphasis is put on learning more than on teaching. The tutorial functions in ILS are focussed on coaching, motivating, giving advice and evaluating the student behavior.

Among the many contributions to the subject, we would like to cite, as closer to our work, the contribution by Vassileva et al. [4] who have built a distributed and collaborative multiagent helping environment where it is possible to get human resources according to request. Canut, Gouraderes and Sanchis [5] use a novel agent model with mobility, autonomy and intelligence for their ITS design. El Alami, and de Arriaga [6] presented also a specific intelligent agent composed by state and control modules, three layers (reactive, tactic and strategic) for the specific knowledge needed by the agent to execute its task, planning and learning capabilities, as well as communication and collaborative functionalities including natural language understanding. Laureano and de Arriaga [7], [8] have proposed a reactive multi-agent architecture without implementing a detailed student model and planning capabilities, useful for coaching purposes.

Another important break-trough has been the introduction of affective computing in ILS. Affective computing, according to Picard can be defined as "computing that relates to, arises from, or deliberately influences emotion" [9]. A large amount of papers in the literature claim that affect plays an extremely important role not only in decision-making but also in human due to affect all cognitive processes [10], [11]. In the past fifteen years a large increase in research directed towards adding affective components to human computer interaction has been produced, as an obvious field of application of this subject [12].

Evidently, addressing those problems is not an easy task, although everybody will recognize that affective sensors and their automatic measurements and interpretations would be quite useful for many areas in different fields of application. However, evaluating and interpreting affective measurements presents a challenge due to many ambiguities related to affect definition, communication, and interpretation.

The questions stated above can aid to classify a great deal of research papers. A first line of research is mainly devoted to the use of sensors to try to elicit human emotions. The work by D' Mello et al. [13] follows this line; they integrate nonintrusive affect-sensing technology with a tutor in an endeavour to classify emotions on the basis of facial expressions, movements and conversational cues. Some other papers try to concentrate on the evaluation of affective interactions [14] mostly related to behaviour-based actions. But a good picture of the state of the art of affective computing and its challenges can be acquired reading the white paper by Picard [15] dealing with many points of the subject including ethics. Affective computing with the help of pedagogic agents has been introduced in ILS by de Arriaga et al. [16] emphasising the obtainment of the most suitable affective and cognitive tactics taking into consideration the real mental state of the student.

Besides, fuzzy logic techniques have also been used not only to face the evaluation problem but also to represent specific parts of the human expert's model and the student's model [17].

As a result of those contributions, the increase of functionality for the last ILS has been tremendous [18]; it represents a challenge for assessing the student learning process and related details. 


\section{THE EVALUATION PROBLEM}

The problem of the students' evaluation has always been a question of concern in Academic Institutions due to its inherent difficulties, associated to the understanding of the students' learning process. In recent years there have been some attempts to improve classical evaluation procedures by including fuzzy logic techniques. Biswas [19] presented a fuzzy evaluation method for the application of fuzzy sets in students' answers evaluation. In the same paper he generalizes his proposal by adopting a fuzzy matrix-valued marking. However, this approach has several drawbacks: because he uses a matching function $\mathrm{S}$ to measure the degrees of similarity between the standard fuzzy sets and the fuzzy marks needing a considerable amount of time to perform the matching operations. Besides, two different fuzzy marks may be translated into the same awarded grade and this seems not very fair. Chen and Lee [20] have also presented two fuzzy methods overcoming Biswas's drawbacks.

However, when we are dealing with Intelligent Learning Systems we have to face more complex evaluation objectives, as we will see later on, and the above mentioned methods are not appropriate.

De Arriaga et al. [21] have proposed two fuzzy evaluation methods to initially assess and update in a continuous way the student learning style, which is an important feature of the student behavior model. In this paper both methods are generalized to be applied with any ability. Some other fuzzy techniques are also here included.

\section{STUDENT'S ASSESSMENT FOR AFFECTIVE AND COGNITIVE COMPUTING}

The student overall behavior, represented by his answers, solutions to the problems and decision making, is the only input to the system for its guidance and functioning. In consequence, student's assessment has to be used for the most important decisions of the system, which obviously depend on its capability. In general, there many things, related to the student's learning behavior and state which have to be continuously evaluated. Here we will refer only the situations related to the most important objectives. They are:

A)-in relationship to the student model:

1-Obtain and update the student learning style. According to Honey [22] and Alonso [23] there are four basic learning styles: theoretical, reflexive, pragmatic and active. Actually any student participates in the four basic styles with greater or lesser intensity. The four of them are adequate for specific purposes although each student uses one of the four learning styles more often because he feels more comfortable with it. This objective attempts to initially obtain the student learning style not only to personalize the learning process according to his mentality, but also to improve the use of the remainder learning styles. As a consequence of these attempts done by the system, the student learning style can change. The evaluation has to give information of the changes introduced into the learning style.

2-Obtain and update the student affective state. According to [16], the OCC model by Ortony, Clore and Collins [24] has been introduced. This model is based on the cognitive theory of emotion and is capable to be implemented computationally. The OCC model provides information on how to build an interpretation of a situation from the user's point of view and the leading interpretation. Emotions appear as a result of an evaluation process called appraisal. The model assumes that the emotions can arise by evaluating three aspects of the world: events, agents, or objects. Events are the way people get things that happen. Agents can be people, animals, inanimate objects or even abstractions, if they can interact. Objects are passive things. The elicitation of an emotion depends on a person's perception of the world, in other words, his/her "construal". If an emotion such as distress is a reaction to some undesirable event, the event must be construed (catalogued) as undesirable. The next step has been the production in our learning environment of a list of possible pedagogical events, and their classification according to the type of emotion that they elicit. As an example of event: the agent can decide to offer some help to the student or, equivalently, the student can ask for help. The student can accept the generic or specific help (gratitude) or not (anger). We have considered up to twenty different pedagogical events including the case when the student gives up the task. The last step has been to fully analyse each pedagogical event as a function of its desirability and intensity variables such as realization and unexpectedness. In some cases the desirability has to be obtained by making an additional question to the student, taking always into account the nature of the student's goal. The realization can be inferred from the grade obtained by the student. The degree of unexpectedness can be estimated by the actual performance of the student.

The result of the analysis of pedagogical events produces a set of production rules assembled in the specific knowledge module of the interface agent. Those production rules make it capable of inferring or eliciting the student's emotions and their intensity for each pedagogic event. Besides, a collection of affective tactics has been devised in order to always motivate the student at any situation.

3- Update the history of the student learning process. Often the student history has been structured into components, as, for example: number of student learning sessions, frequency of sessions, session duration, number of errors and type of error for each session, number of exercises and types in each session, etc. Those components, updated after ending each learning session, are used for many different purposes; the evaluation process has to obtain those components extracting them from the usual interaction of the student with the system.

4- Update the history of the student affective states. It is very simple once the different student's affective states along the time have been obtained.

5- Update the student fuzzy conceptual graph. The student model includes a fuzzy conceptual graph of the learning domain, detailing the graph nodes (concepts, properties, procedures, etc...) acquired and mastered by the student as well as the remainder nodes not yet acquired. The acquired nodes have adjoined a fuzzy set showing the learning intensity of those concepts, properties, etc. That intensity that changes with time, will have an impact on its suitable use by the student.

6-Obtain regularly the student cognitive components of the ability to be learnt, as they are acquired by the student. This objective could be assimilated to the one described in the previous paragraph, but here we want to emphasize the obtainment of all the expert cognitive components of the ability to be learnt, including the human expert mental 
models. BCTA (Behavioral-Cognitive Task Analysis) is a methodology based on the knowledge elicitation techniques [25], [26] that allow the decomposition of the ability to be learnt or the problem to be solved, into its cognitive components considering three levels of execution of the ability:

a) A first approach to get familiarised with the task.

b) Regular execution of the task and

c) Expert execution of the ability or task.

The obtained cognitive components can be classified into: facts, declarative knowledge (concepts, relationships, properties), procedures, strategies and mental models. By following some of the steps of this methodology, the system is able to obtain the student cognitive components acquired.

7-Obtain the strategies followed by the student in the solution of the problems, inferred from the student solution path. The evaluation has to have suitable models for that inference.

8-Update the potential basic conceptual learning abilities acquired by the student and contained in the student model. First, we have to consider which of those basic conceptual learning abilities are relevant to the task or ability to be learnt. Usually the basic conceptual abilities, such as: geometrical reasoning, arithmetic reasoning, spatial vision, linguistic reasoning, analogue reasoning, etc., are too elementary to be considered as cognitive components in previous objectives included above.

9-Obtain the student peer-review by means of cooperative actions of the learning group. The peer-review will also integrate the student module. It will be used to check (when possible) the inferences done by the system when analysing the student responses.

B)-in relationship to the expert module or to the agents in charge of the different aspects or ingredients of the human expert domain:

1-Obtain the student errors as difference between the expert and student behaviors and analyse elementary those errors and their shallow reasons.

2-Obtain the domain graph nodes related to the errors and the cognitive components which have not been acquired through learning.

3-Obtain, using also the student history, the profound causes of error, and infer the related graph nodes to start with the remedial tactics.

C)-in relationship to the tutor module or to the agents in charge of the different tutorial functions:

1- The arbitration of different agents wishing to act at the same moment along the learning process.

2- The suitable intervention of the agent in charge of the functions of General Didactics. This agent monitors and operates in relationship to general learning aspects and situations of the student mental state such as: motivation, interest, tiredness, fatigue, etc. This agent can even suggest the interruption or closing of the learning session when some negative aspects are present.

3- The intervention of tutors in charge of the domain microworlds to possibly notify the errors to the student and propose different remedial actions. In the case when the student is advancing quite well along the learning process without significant errors, the tutors could propose proactions in order to improve or accelerate the learning process.

4- The determination of the final or global student' evaluation and the linear ordering of the group of students.

D)-in relationship to the interface:

1-The personalization of the whole interface or part of it (some of its windows) in order to adapt the interchange of information between the student and the system to the student learning style and the particular student mental state.

\section{FUZZY TECHNIQUES}

Here are some of the fuzzy techniques that have been installed in NEOCAMPUS2. They can be used in several contexts or objectives already detailed.

A) Initial evaluation of an ability style (before starting the learning process). Probably the most widely used is the following technique. It can be decomposed into the following steps:

1-The initial questionnaire for the initial student's ability style (and also the following ones used for control or evaluation purposes) is treated previously, before using it, in order to adjoin a fuzzy set to each item in the questionnaire. This fuzzy set qualifies the item in terms of the basic ability styles; so, for example, item 2.16 could be described as:

\section{$\mathrm{F}(2.16)=0.45 /$ style $1+0.95 /$ style $2+0.05 /$ style $3+0.2 /$ style 4}

meaning that the intensity level of the basic ability styles (style1, style2, style3, style4) needed to solve or answer correctly item 2.16 is, respectively, $(0.45,0.95,0.05,0.2)$.

2- Student answers are also graded in the same way, obtaining a fuzzy set $\mathrm{R}(2.16)$ associated to each item response. So, if the student's answer is completely correct, as in the case of guessing the right answer to a multiple choice question, $\mathrm{R}(2.16)$ will be identical to $\mathrm{F}(2.16)$. If the student has to construct the answer, that answer has to be estimated in terms of the basic ability styles or, what is easier, it is estimated in terms of the regular linguistic terms: excellent, very good, good, etc. These linguistic terms have been previously converted into fuzzy sets, according to the nature of the item. In this case the real estimation of the student's answer will be the fuzzy composition of two fuzzy sets: the set associated to the item and the set associated to the linguistic term. That is to say, in our example:

$$
\mathrm{R}(2.16)=\mathrm{F}(2.16) * \mathrm{GOOD}
$$

Whereas * means the fuzzy composition of those two fuzzy sets; GOOD is the fuzzy set associated to this linguistic term.

3-After estimating all the student's responses, now we can build for this student (James) the fuzzy sets: Style1(James), Style2(James), Style3(James) and Style4(James), defined on the universal set $\{0,0.1,0.2,0.3, \ldots, 0.9,1\}$ as follows:

$$
\text { Style } 1(\text { James })=\mathrm{t} 1 / 0.1+\mathrm{t} 2 / 0.2+\mathrm{t} 3 / 0.3+\ldots . .+\mathrm{t} 10 / 1
$$

The values $\mathrm{t} 1, \mathrm{t} 2, \mathrm{t} 3, \ldots, \mathrm{t} 10$ are obtained only from the terms tx/style 1 existing in $\mathrm{F}$ (item) and $\mathrm{R}$ (item). The procedure can be illustrated by means of an example:

Suppose we want to obtain $\mathrm{t} 3$ (the pertaining level of 0.3 in Style1(James)); we will look for all items $\mathrm{F}(\mathrm{X})$ containing the term $0.3 /$ style 1 . For each item of those founded we will attribute to $\mathrm{t} 3$ one point (1.0) when the answer is fully correct; if not, we will attribute to $\mathrm{t} 3$ only a fraction of a point 
(according to the estimation R(item) of the student's answer). The sum of the points attributed to $\mathrm{t} 3$, divided by the number of times that 0.3 has appeared in the fuzzy sets of all the items in the questionnaire is the final value for $\mathrm{t} 3$.

\section{B) Permanent updating of an ability style.}

The student's ability style is not static, but changes relatively along the learning process, needing a continuous update. This updating or the initial assessment, if it has not been possible to get it from the beginning, can be done by applying a similar procedure to the questions proposed to the student during his/her interactions with the system.

In any case, and according to the actual student's ability style, determined by the four fuzzy sets Style1, Style2, Style3, Style4, the ITS agent in charge of the tutorial function or general didactics, will establish by means of a fuzzy controller the appropriate actions for advice or proaction. These actions will be obtained by means of fuzzy rules whose antecedents will contain the ideal fuzzy sets Style1, Style2, Style3, Style4 obtained only from $\mathrm{F}(\mathrm{x} . \mathrm{yz})$ which will interact with the student sets Style1(James), Style2(James), Style3(James), and Style4(James), through the fuzzy mechanism known as "fuzzy modus ponens".

C) Obtainment of the student's cognitive components of the ability to be learnt, by means of BCTA (BehaviouralCognitive Task Analysis) methodology. For that purpose we will follow the following steps:

1-According to this decomposition, all the questions, problems and projects proposed students during the learning process and controls, are characterised by means of a fuzzy sets $\mathrm{C}$ on the universe of the cognitive components in a similar as we did in a) with the sets F(item)

2-The student answers and solutions are also estimated, in a direct way by analysing the cognitive components of the answer, or indirectly, by using linguistic terms (excellent, very good, good, etc.) which also have fuzzy sets associated to them. In this last case, the composition of $\mathrm{C}$ and the fuzzy set of the appropriate linguistic term provide the fuzzy estimation of the student's answer in terms of the cognitive components of the ability to be learnt.

3-The acquired cognitive components are estimated by fuzzy union of the estimation of the student's response. That way we get a cognitive fuzzy graph detailing the student leaning level for each cognitive component.

4-A very important point of this procedure is the obtainment of the student mental models, which according to the literature are very different from those of the human expert. The student mental models are used, outside the evaluation process to try to show to the student, by means of practical questions and problems, that they are insufficient and no adequate for reasoning within the learning domain.

D) Fuzzy student's evaluation.

Student learning is a long process highlighted by a set of errors. In parallel, the ITS has been working on error detection and remedial tactics offered to the student to fix them up. Also in parallel or at the end of the learning process an evaluation or global estimation of learning has to be done.

One of the main tendencies for the ITS construction is the development of the genetic graph corresponding to the knowledge of the learning domain. We use to enhance the genetic graph with the declarative knowledge of the learning ability, the tactics and procedures necessary for the acquisition of the main ability and its sub-abilities, the functioning strategies for this ability and even the human expert mental models. We do that by means of BCTA, already mentioned, able to get the cognitive and behavioural components for the execution of a human task. As we expressed before, all the questions, problems and projects proposed to the student within the evaluation process can be characterised by means of a fuzzy set defined on the universe of the cognitive components of the learning ability. In the same sense, the student answers can be fuzzily estimated as a function of those cognitive components for the main ability and its sub-abilities, as we did in c).

The aggregation of all the student answers allows to obtain a cognitive fuzzy graph detailing the student learning level for each cognitive component. If needed, it is possible and easy to defuzzify the graph by means of another fuzzy operation of aggregation to reach a quantitative global evaluation for the student or a fuzzy set which can be translated linguistically to the usual qualitative level such as: good, very good, and so on

\section{DYNAMICS2: RESULTS AND DISCUSSION}

NEOCAMPUS2 is the new version of a multi-agent environment for educational research and applications [27] [28] including the production of ILS. It is a long-range international effort aiming at different practical and research goals trying to cover many aspects related to the learning process and their computer implementation.

Due to the variety of possible E-L scenarios and their features, the main functions contemplated for their applications, including web-based tutors are the following:

-track, manage and update the student log history including all the significant details: number of sessions, frequency, duration, number and time of different errors, etc.

-analyze the students' (and students group) errors by obtaining shallow and deep error reasons related to scientific knowledge or expert experience;

-obtain from the user's interaction the constituents of the student model and its parameters such as: learning style, interest, effort, attention, capability, etc.

-tutorial help in its different interpretations: advice, guidance, tips, remedial tactics, instructional design, evaluation, coaching exercises, etc., by using the cognitive elements of the ability to be learnt;

-personalize the operation of the system, including its interface, to adapt it to the particular student features;

-group guidance and help for the obtainment of the project group structure integrated by a set of roles and profiles; -monitor the student group structure by tracking each role and their integration in the group;

-evaluation (with different purposes) of each individual behavior and of the whole group;

-monitor and enhance personal and group motivation;

-manage and control simulators and tools for decision making, evaluation or problem solution;

-update Knowledge Bases with Internet information, duly classified and summarized;

-promote group functioning, interaction and operation aiming at cooperative and collaborative learning

-manage and control Knowledge Bases and Experience Systems.

DYNAMICS2 is a NEOCAMPUS2 spin-off ITS, devoted to systems modelling in the state-space, allowing interactive, collaborative and cooperative decisions made by a team of 
students. A full conceptual graph enriched with methods, tools and procedures, as well as semantic and pragmatic information describes the domain scientific knowledge. The graph has been initially designed and enriched with different sources of information: books, journals, news and Internet information. The human expert experience management system has also been built in by applying the BCTA technique to a team of human experts. The experience management model (EMM) used in this case [29] relates the different tasks involved in experience management. It is composed by a knowledge kernel and two shells around it. The knowledge kernel contains not only the experience data base but also the use-related knowledge including the vocabulary. The next shell around the kernel consists of the problem solving cycle including: the problem acquisition, experience evaluation and retrieval, experience adaptation, and experience presentation. The outer shell of the EMM contains the development and maintenance methodology. The knowledge kernel and the problem solving cycle are the subject of the development and maintenance methodology. The diverse processes that occur in this shell address the acquisition and maintenance of the knowledge in the kernel as well as the technical, organizational and also managerial aspects of the problem solving cycle and its implementation. Those processes are also integrating a cycle which must be started each time a kind of maintenance is required. The two cycles already mentioned present significant differences:

1) The problem solving cycle supports complex problem solving by providing appropriate experience. It contains several elements for complex problem identification; problem acquisition module; experience evaluation and retrieval; experience adaptation; and the presentation module.

2)The Development and Maintenance Methodology cycle includes several processes in order to acquire and update the required experience knowledge and to customize the problem solving cycle, and requires modelling and maintenance of the experience kernel.

The main aspects covered by this last cycle are the following:

a) The process of project management, including cost and resource assessment, time schedules, project plans, quality control procedures, etc.

b) The specification of the different kinds of products or deliverables that must be produced.

c) The process of product development and maintenance, including all technical tasks that are involved in the development and maintenance of the software.

d) The analysis and organization of the environment in which the system should be introduced.

DYNAMICS2 also integrates affective and cognitive computing within the following operational cycle:

A) According to the user's needs for learning a specific topic within a learning environment, the system tentatively designs the corresponding "global learning element". The systems situates the practical objectives of the user on the learning domain graph and the human expert experience management system previously built in the ILS, evaluating all the nodes and arcs needed to obtain the user's learning objectives in terms of declarative knowledge, tactics and strategies to be learned, as well as the collection of exercises to be conducted. If the learning element fulfils all the other requirements of the user, such as learning duration, possession of the initial knowledge to start the learning, etc., that learning element is adopted. Should the situation prevent the system to adopt a learning element, a message is sent to the user with the limitations so far founded.

B) The system can guide the complete learning process (by means of a control agent) and also the student can assume when he/she wants the conduction of the process.

C) At any intervention of the student the control agent sets the analysis (shallow and deep) of the user's intervention. Shallow analysis determines the possibility of errors and their type, by comparing the student's with the human expert behaviors. At the same time the student's state (including affective features) is updated and according to their results the control agent allows the intervention of the agents in charge of General Didactics to establish the intervention of pedagogical agents and general emotive actions. Then the control agent transfers the control to the agents in charge of the different domain micro-worlds to define cognitive tactics and remedial procedures. Then the control establishes the ordered actuation of the agents, usually starting with pedagogic agents, by means of a fuzzy control that integrates cognitive and affective computing.

D) Once the student has completed the exercises or remedial tactics imposed, a new analysis is done to check those tactics and remedies. If the evaluation is all right the learning process continues.

In the case of a full team of students trying to solve cooperatively a problem, the solution can be split in different tasks which can be assumed individually, although their execution is first discussed by the whole group. Some other times the full group can work together on a single execution or problem to choose the best solution or integrate several ones.

Each member of the group has also to evaluate the actions taken by the remainder members (peer-review), the results of which are used for the system to check the inferences made by the system itself. The decisions made by the team are discussed previously and afterwards when the results of the decisions become noticeable. The system is now capable of finding the students' mental models; the differences of those mental models with those of the human expert and the affective situations of the members of the group trigger tutorial affective and cognitive actions to gradually reduce the found differences. NEOCAMPUS2 has been provided with the fuzzy logic techniques described above, which have been inherited by DYNAMICS2.

The evaluations made by the systems working with 8 experimental groups of 20 students have been compared to those obtained by 8 instructors (working together). They have also analysed the cognitive components and conceptual graphs obtained by the system. The evaluations have been used in the following contexts:

1-to check if the information provided guarantees the proper functioning and arbitration of the agents in charge of the domain micro-worlds and of the General Didactics;

2-to check if the fuzzy techniques used in the evaluations give plausible results according to traditional academic experience;

3-to check if the student's cognitive fuzzy graph conveys enough information to trigger and establish suitable tutorial tactics by the agents in charge of those tasks.

The final report of the instructor is very positive. It shows full agreement (91\%) with the results obtained by the system. 
The results obtained with the experimental groups have also been compared with those obtained in 8 control groups (20 students each) which did not use the system, but traditional learning exercises. The experimental groups show $24 \%$ better grades than the control groups.

\section{CONCLUSIONS}

1-The results so far obtained with DYNAMICS2 show that the described procedures allow the assessment of the cognitive components of the task and sub-tasks of the learning domain and the global and partial student's evaluation. The use of the mental models of the human expert reduces the time of the students to become expert, quite significantly (27\% obtained in specific tests)

2-The obtainment of the particular ability styles for each group member allows the personalization of the interface without preventing full collaboration of the student with the rest of the group.

3-Affective computing, although it is a complex task with a long way of future development, is an important subject from the educational point of view. The affective mood of the student can influence not only his learning process but also his way of thinking; in consequence it should be considered in Intelligent E-Learning Systems.

4-Emotional data can be obtained from the student by different methods including specific sensors, but the student's behavioural actions and reactions with the system can provide sufficient data for, at least, a first approach to infer the student's emotions and interact with them.

5-The results obtained with AFFECTION, the affective platform specially designed, applied to DYNAMICS2, although so far limited, seems to show, once more, promising aspects concerning the benefits of affective computing for human learning. Those improvements are, at least, related to grades, satisfaction and finishing tasks.

6-The use of fuzzy sets, to specify the relevance or learning intensity of cognitive elements, and fuzzy rules that establish or modify those fuzzy sets, is not an important drawback for the application of those guidelines to systems that instead of providing a single number as the student's assessment, yield a complex fuzzy model.

\section{REFERENCES}

[1] de Arriaga, F., El Alami, M., Ugena, A., 2004, Aprendizaje Centrado en el Estudiante: Aprendizaje por Proyectos, in Psicología Educacional, ed: Sandra Castañeda, México.

[2] de Arriaga F., Arriaga A., El Alami M., 2005, Multi-Agent Platform for Educational Research in Intelligent ELearning Systems, Journal of Advanced Technology on Education, Vol. 1 (4), pp. 150-155.

[3] El Alami, M., de Arriaga, F., 2004, Aprendizaje Centrado en el Estudiante: Aprendizaje por Problemas, in Psicología Educacional, Ed: Sandra Castañeda, México.

[4] Vassileva, J., Geer J., et al., 1999, A Multi-Agent Design for Peer-Help Environment, Artificial Intelligence in Education, eds: S.P. Lajoie, M Vivet, pp. 38-45, IOS Press.

[5] Canut, M., Gouarderes, F., Sanchis, E., 1999, The Systemion: A New Agent Model to Design Intelligent Tutoring Systems, Artificial Intelligence in Education, Eds: S.P. Lajoie, M. Vivet, pp. 54-63, IOS Press.
[6] El Alami, M., de Arriaga, F., Ugena, A., 1999, SIMUL: An Intelligent Simulation Environment for Interdisciplinary Projects, in Project Studies, ed: O. Olensen, Roskilde University Press.

[7] Laureano, A. L., de Arriaga, F., 1998, Multi-Agent Architecture for Intelligent Tutoring Systems, Interactive Learning Environments, 6, pp. 225-250.

[8] Laureano, A. L., de Arriaga, F., 2000, Reactive Agent Design for Intelligent Tutoring Systems, Cybernetics and Systems, 31, pp. 1-47.

[9] Picard, R. W., 1997, Affective computing. The MIT Press, Cambridge MA.

[10] Kinard, E. M., 2001, Perceived and actual academic competence in maltreated children. Child Abuse and Neglect, Vol. 25, 1, pp. 33-45.

[11] LeDoux, J., 1998, The emotional brain: The mysterious underpinnings of emotional life. Weidenfeld and Nicholson, London.

[12] Panitc, M., 2005, Affective Comnputing, Idea Group Inc.

[13] D'Mello et al., 2005, Integrating Affect Sensors in an Intelligent Tutoring System, Proceedings International Conference on Affect Computing and Intelligent Interaction, Beijing, Springer Verlag.

[14] Picard, R. W., Daily, S. B., 2005, Evaluating Affective Interactions: Alternatives to asking what users feel, Proceedings International Conference on Affect Computing and Intelligent Interaction, Beijing, Springer Verlag.

[15] Picard, R. W., 2003, Affective Computing: Challenges, White Paper, MIT Technical Report.

[16] de Arriaga, F., El Alami, M., 2006, Affective Computing and Intelligent E-Learning Systems, Proceedings IADAT International Conference on Education e-2006, Barcelona, pp. 115-120.

[17] de Arriaga, F., Gingell, C., Arriaga, A., Arriaga, J., Arriaga, F. Jr., 2008, A General Student's Model for Intelligent E-Leaning Systems, Proceedings ECC'08, $2^{\text {nd }}$ European Computing Conference, Malta

[18] Arriaga, A., El Alami, M., de Arriaga, F., 2003, NEOCAMPUS2: New Trends in the Functionality of Intelligent Learning Systems, Proceedings IASTED International Conference on Artificial Intelligence. Benalmadena, pp. 380-386.

[19] Biswas, R., 1995, An Application of Fuzzy Sets in Students' Evaluation, Fuzzy Sets and Systems, 74, pp. 187-194.

[20] Chen, S., Lee, Ch., 1999, New Methods for Students' Evaluation Using Fuzzy Sets, Fuzzy Sets and Systems, 104, pp. 209-218.

[21] de Arriaga F., Laureano A.L., El Alami M., Arriaga A., 2002, Some Applications of Fuzzy Logic to Intelligent Tutoring Systems, in Educational Technology, Vol. 2, pp. 1222-1227.

[22] Honey, P., 1986, Using our Learning Styles, Ed: A. Mumford.

[23] Alonso, C., Gallego, D., 1994, Estilos de Aprendizaje, Ed. Mensajero. 
[24] Ortony, A., Clore, G., Collins, A., 1988, The Cognitive Structure of Emotions, Cambridge, UK, Cambridge University Press.

[25] El Alami, M, de Arriaga, F., 2013, Intelligent E-Learning Systems and the Transfer of Novices into Experts, International Journal of Computer Applications, Vol. 82, $\mathrm{n}^{\circ} 8$, Novembre, pp. 44-54.

[26] A. L. Laureano, F. de Arriaga, M. García-Alegre, 2001, Cognitive Task Analysis: A Proposal to Model Reactive Behaviours, Journal of Experimental and Theoretical Artificial Intelligence, 13, pp. 227-239.
[27] de Arriaga, F., El Alami, M., 2004, NEOCAMPUS2: A Multi-Agent Environment for Educational Research and Applications", in Innovation, Technology and Research in Education, Iadat, pp. 194-199.

[28] El Alami, M., de Arriaga, F., 2003, Web-Based Tutors for Collaborative E-Learning, in Advances in TechnlogyBased Education, Vol. III, Formatex, pp. 2009-2014.

[29] de Arriaga, F., Arriaga, A., El Alami1, M., 2006, Experience Management: Models and Implementation, in Current Developments in Technology-Assisted Education, Formatex, pp. 841-849. 